\title{
CONSULENZA CONTABILE AMMINISTRATIVA: UN NUOVO MODO DI GUIDARE UN'AZIENDA ATTRAVERSO LA TECNOLOGIA
}

\section{ARTICOLO ORIGINALE}

SUMAR, Ramiro Rodrigues ${ }^{1}$

SUMAR, Ramiro Rodrigues. Consulenza contabile amministrativa: un nuovo modo di fare impresa attraverso la tecnologia. Revista Científica Multidisciplinar Núcleo do Conhecimento. Anno 06, Ed. 07, Vol. 12, pp. 91-104. Luglio 2021. ISSN: 2448-0959, Link di accesso: https://www.nucleodoconhecimento.com.br/economiaaziendale/attraverso-la-tecnologia, DOI: 10.32749/nucleodoconhecimento.com.br/economia-aziendale/attraverso-latecnologia

\section{RIEPILOGO}

Obiettivo: Descrivere l'impatto degli ostacoli e delle potenzialità che la tecnologia ha portato alla consulenza contabile. Domanda: In che modo la consulenza contabile amministrativa può aiutare un'azienda a utilizzare le tecnologie? Metodi: Lo studio è una rassegna bibliografica. È stata condotta una ricerca con i descrittori: tecnologia; innovazione; contabilità; sulla piattaforma dati di Spell (Scientific Periodicals Electronic Library) nel giugno 2021. La perquisizione ha portato a 10 articoli. Risultati: i risultati portati come ostacoli: II crescente progresso tecnologico, adattarsi all'uso di queste nuove tecnologie, Centralità del ruolo del contabile nei modelli tradizionali, Mancanza di conoscenza del software,Mancanza di forza lavoro specializzata, malfunzionamento della connessione, manodopera qualificata. E come potenzialità: Utilizzo di diverse tecnologie digitali, Agilità e guadagno di tempo, Convenienza, rapporto inseparabile tra tecnologia e contabilità consultiva

\footnotetext{
${ }^{1}$ Laurea magistrale in Controllo, Specialista in Gestione Tecnologica, Amministrazione Strategica, Sicurezza delle Informazioni e Laureato in Scienze Contabili.
}

RC: 93887

Disponível em: https://www.nucleodoconhecimento.com.br/economia- 
promuovendo un supporto agile e di qualità ai suoi vari utenti, soddisfazione del cliente, velocità e flessibilità e riduzione dei costi. Conclusioni: È chiaro che le tecnologie dell'informazione influenzano positivamente l'esercizio della contabilità consultiva, anche se oltre ai vantaggi presenta i suoi svantaggi. Vale la pena ricordare che le tecnologie consentono il controllo delle informazioni contabili per l'ingresso e l'uscita di materiali e input, nonché la loro origine e destinazione finale, evitando perdite inutili, tra cui deviazioni, agilità, flessibilità e sicurezza.

Parole chiave: Consulenza, Innovazione, Mezzi Tecnologici, Sicurezza dell'Informazione.

\section{INTRODUZIONE}

Il legame tra contabilità e tecnologie si è perpetuato per decenni, poiché, oltre ad essere scienza, la contabilità è uno dei sistemi informativi utilizzati nella gestione aziendale più vecchia, il che rende diversa la contabilità del passato e la contabilità del presente sono i modi in cui portano alla gestione aziendale (FERREIRA et al., 2017).

La tecnologia è procedono nel corso degli anni, innovando in modo da soddisfare il bisogno dell'essere umano. Pertanto, essendo uno strumento illimitato, l'evoluzione delle tecnologie combinate con la contabilità porta contributi rilevanti alla crescita delle organizzazioni (SILVA et al., 2020).

L'uso continuo delle reti informatiche e la combinazione di vari strumenti contabili favoriscono la memorizzazione, la registrazione, l'elaborazione e facilitano la realizzazione dell'incrocio delle informazioni sempre più velocemente, il che consente un processo decisionale più sicuro all'interno dell'organizzazione (MARION; RIBEIRO, 2017; DE ANDRADE SIMÕES et al., 2021).

RC: 93887

Disponível em: https://www.nucleodoconhecimento.com.br/economia- 
Attualmente, la ricerca di informazioni è stata utile e allo stesso tempo rilevante per la crescita dell'organizzazione. II trasferimento di informazioni alla conoscenza, così come la tempestiva applicazione delle conoscenze nella pratica e la distribuzione di questi atti per lo sviluppo di un'azienda, sono potenzialità della tecnologia a favore della contabilità consultiva per l'imprenditore.

Tuttavia, molti contabili non usano questo strumento come un modo per guadagnare spazio, tempo e trattenere i propri clienti, chiarendo che in alcune situazioni, le tecnologie per i contatori non vengono utilizzate. In questo collocamento, identificando la problematizzazione dello studio in questione, c'è la domanda che ha guidato il tema di questo studio: In che modo la consulenza contabile amministrativa può aiutare un'azienda a utilizzare le tecnologie?

L'attuale mercato del lavoro spesso motiva le persone a riformularsi nelle loro attività professionali, perché il sistema di istruzione universitaria di base e le nuove conoscenze diventano ancora pochi in considerazione della pratica che lo studente eseguirà dopo la laurea.

Secondo Villa et al (2012, p.107), "i commercialisti tradizionali hanno l'opinione che gli imprenditori non cerchino le conoscenze necessarie per gestire la propria attività, cioè non attraverso corsi di miglioramento o formazione in amministrazione". Per parafrasare questa citazione dell'autore, indica che l'imprenditore ha solo l'interesse a soddisfare le richieste di tasse, dimenticando così il mercato competitivo, che fa perdere spazio all'azienda e non svilupparsi nella sua qualità.

In questo collocamento, si osserva l'importanza del contatore consultivo. Questo lavoro si aggiunge alla giustificazione del ricercatore di capire perché molti commercialisti non utilizzano la contabilità consultiva per lavorare con i loro clienti, poiché questo strumento di aiuto soprattutto nei tempi pandemici del nuovo coronavirus esercita influenza in modo che l'azienda non sia a rischio di avere impegno nel suo reddito nel mercato competitivo.

RC: 93887

Disponível em: https://www.nucleodoconhecimento.com.br/economia- 
Pertanto, il tema diventa rilevante, perché con l'avvento delle tecnologie, la contabilità delle aziende ha preso una direzione diversa dalla contabilità decenni fa e con questo, il commercialista ha ora la responsabilità e l'impegno di rendere la sua professione identificata dalla società e dagli imprenditori come una professione che sta avanzando per adattarsi ai mezzi tecnologici al fine di garantire maggiore sicurezza e affidabilità alle organizzazioni.

Pertanto, questo lavoro ha come obiettivo generale: descrivere l'impatto degli ostacoli e delle potenzialità che la tecnologia ha portato alla consulenza contabile.

\section{PROCESSO METODOLOGICO}

Questo studio è una rassegna bibliografica sistematica di natura qualitativa. Secondo Sampaio e Mancini (2007) questo tipo di studio viene effettuato attraverso la ricerca di articoli rilevanti per il tema ricercato, basati sulla sintesi di soggetti rilevanti abbinati a metodi di indagine sistematizzati, analisi critica, sintesi delle informazioni scelte e problemi importanti che richiedono ulteriori studi.

Per svolgere la ricerca sono stati seguiti i seguenti passaggi raccomandati da Sampaio e Mancini (2007): Formulazione della domanda guida dello studio; Ubicazione degli studi; Valutazione critica degli studi; Raccolta dei dati; Analisi dei dati; Aspetti etici.

Dalla costruzione di una domanda guida emergono alcune ipotesi che guidano e/o delimitano questo studio, consentendo così di evidenziare l'applicabilità delle tecnologie nell'universo della consultazione dei numeri e dei valori a cui è inserito il commercialista. La domanda che ha guidato l'argomento era: in che modo la consulenza contabile amministrativa può aiutare un'azienda a utilizzare le tecnologie?

RC: 93887

Disponível em: https://www.nucleodoconhecimento.com.br/economia- 
II materiale è stato selezionato nelle banche dati: Spell (Scientific Periodicals Electronic Library). II campione di studio consisteva in articoli (ricerca originale; articoli di revisione; monografie, tesi e tesi). Gli articoli in portoghese e inglese sono stati utilizzati come criteri di inclusione, dal 2016 al 2020 che sono disponibili per intero e gratuitamente. Tutti gli articoli che sono stati inquadrati in questi criteri, che nel loro settore delle conoscenze coprivano la contabilità e avevano come titolo o argomenti astratti rilevanti per l'uso delle tecnologie sono stati inquadrati per la ricerca. Altri articoli che non sono stati inclusi o duplicati sono stati esclusi. Le parole chiave utilizzate per cercare questi articoli in Spell erano: tecnologia; innovazione; contabilità; contabilità consultiva.

La valutazione critica delle produzioni selezionate e incluse nello studio ha dato luogo a interpretazioni e sintesi dei risultati, identificando le informazioni scientifiche presenti in ogni studio e i fattori rilevanti per lo studio in questione suggerendo linee guida per la ricerca futura. Per convalidare lo studio, il ricercatore chiarisce quali lacune sono state trovate nello studio e quali percorsi futuri altri ricercatori possono adottare nella loro ricerca scientifica. Le principali informazioni sono state disposte in tabelle, chiarendo le informazioni pertinenti ai risultati degli articoli, comfaendo le materie con la letteratura pertinente all'argomento.

I dati sono stati raccolti nel giugno 2021. La raccolta è stata eseguita includendo i criteri di inclusione ed esclusione degli articoli. Inoltre, sottolineando che la ricerca cerca anche, sulla base dell'analisi effettuata, di riferire sulla contabilità consultiva e sull'uso delle tecnologie. Sono state effettuate due ricerche intervallate dalle parole chiave.

I dati trovati nella letteratura pubblicata sono avvenuti secondo la proposta di Ercole; Melo e Alcoforado (2014), in cui il revisore dovrebbe cercare di valutare i risultati in modo imparziale, cercando spiegazioni per i diversi o contrastanti risultati nei diversi studi.

RC: 93887

Disponível em: https://www.nucleodoconhecimento.com.br/economia- 


\section{ANALISI DEI RISULTATI E DELLE DISCUSSIONI}

Le ricerche hanno portato a un totale di 343 risultati con le parole chiave. Tutti i titoli e gli abstract sono stati letti e sono stati rimossi gli articoli che potevano rispondere agli obiettivi dello studio in questione, dando luogo a 10 studi.

Pertanto, presentiamo un quadro con il titolo dell'articolo, autori, anno di pubblicazione (Riferimento), obiettivo dello studio e risultati trovati.

Tabella 1 - Articoli selezionati

\begin{tabular}{|c|c|c|}
\hline colo & Obiettivi & dltati \\
\hline $\begin{array}{l}\text { No caminho da } \\
\text { inovação: análise das } \\
\text { capacidades de } \\
\text { inovação de empresas } \\
\text { contábeis diante das } \\
\text { tecnologias digitais }\end{array}$ & $\begin{array}{l}\text { Esaminare la capacità di } \\
\text { innovazione degli uffici } \\
\text { contabili nel mercato } \\
\text { brasiliano, nell'uso delle } \\
\text { tecnologie digitali, in base } \\
\text { alle capacità di sviluppo } \\
\text { tecnologico, } \\
\text { funzionamento, gestione } \\
\text { e transazione. }\end{array}$ & $\begin{array}{l}\text { La contabilità inizia nel } \\
\text { tradizionale percorso di } \\
\text { innovazione digitale, } \\
\text { dimostrando la qualità e il } \\
\text { valore che le soluzioni } \\
\text { tecnologiche possono generare } \\
\text { se sfruttate nel business e } \\
\text { soprattutto nei processi.Questo } \\
\text { aumento della tecnologia } \\
\text { provoca cambiamenti nei } \\
\text { modelli di business contabili. }\end{array}$ \\
\hline $\begin{array}{l}\text { Indústria } 4.0 \text { e avanços } \\
\text { tecnológicos da área } \\
\text { contábil: } \\
\text { percepções } \\
\text { expectativas } \\
\text { profissionais }\end{array}$ & $\begin{array}{l}\text { Analizzare il profilo del } \\
\text { professionista contabile di } \\
\text { fronte ai progressi } \\
\text { tecnologici, con l'obiettivo } \\
\text { di esplorare come si } \\
\text { comporta in relazione alle }\end{array}$ & $\begin{array}{l}\text { La maggior parte dei } \\
\text { professionisti è interessata ad } \\
\text { adattarsi alle nuove tecnologie } \\
\text { per soddisfare i requisiti della } \\
\text { funzione. Tuttavia, ci sono } \\
\text { state alcune resistenze ai }\end{array}$ \\
\hline
\end{tabular}

RC: 93887

Disponível em: https://www.nucleodoconhecimento.com.br/economia- 
Saverio; Carraro e nuove tendenze.

Rodrigues (2020).

Diferentes Modelos de Negócios Contábeis em Relação às Áreas da Contabilidade

Schiavi e Behr (2020). servizi
Características

cambiamenti, specialmente tra i professionisti sotto i 35 anni e nell'area aziendale.

os Identificare il valore L'area contabile focalizzata su consegnato al cliente dei Information Systems ha le sue contabili, consegne basate sulla consulenza nell'elaborazione dei dati, nella tecnologia le dell'informazione e nei sistemi strutturale caratteristiche dei modelli operativi.

di business tradizionali, in relazione alle rispettive aree di attività contabile.

As Contribuições da Controlla i contributi $\mathrm{Si}$ è constatato che il percepire Contabilidade

Consultiva: Um Estudo de Caso/The promossi dal progresso della tecnologia nella consultiva nello scenario Contributions of contabilità consultiva.

Advisory Accounting: A Case Study

Angelim e Barreto (2020).

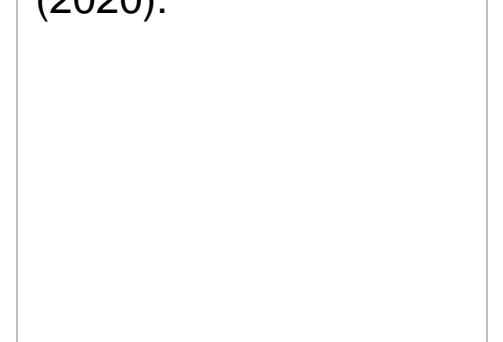

Impact of information technology

nella attuale e il ruolo essenziale della tecnologia nella professione contabile. Le sfide affrontate dai intervistati comprendono l'adattamento all'uso di nuovi strumenti tecnologici, la necessità di delineare strategie per servire meglio il cliente e la necessità di conoscere la gestione e il business di ciascun cliente.
Determinare

on della l'impatto I risultati della ricerca hanno tecnologia dimostrato che la tecnologia

RC: 93887

Disponível em: https://www.nucleodoconhecimento.com.br/economia- 


\begin{tabular}{|c|c|c|}
\hline $\begin{array}{l}\text { accounting line of } \\
\text { works } \\
\text { Asuquo; Dan ed } \\
\text { Effiong (2020). }\end{array}$ & $\begin{array}{l}\text { dell'informazione sulla } \\
\text { linea contabile dei lavori } \\
\text { nel sistema globale. }\end{array}$ & $\begin{array}{l}\text { dell'informazione } \\
\text { un'influenza sostanziale sulla } \\
\text { linea di lavoro contabile. }\end{array}$ \\
\hline $\begin{array}{l}\text { The impact of } \\
\text { information and } \\
\text { communication } \\
\text { technology (ICT) in the } \\
\text { accounting system: } \\
\text { advantages, } \\
\text { applications, } \\
\text { challenges } \\
\text { Al-Shafeay; Al_Dujaili } \\
\text { e Al-Wattar (2020). }\end{array}$ & $\begin{array}{lr}\text { Esaminare } & \text { i vantaggi } \\
\text { delle } & \text { applicazioni } \\
\text { informatiche } & \text { nella } \\
\text { contabilità. } & \end{array}$ & $\begin{array}{l}\text { La contabilità, come il } \\
\text { linguaggio delle imprese e il } \\
\text { sistema informativo, deve } \\
\text { adattarsi alle nuove tecnologie } \\
\text { per servire gli utenti } \\
\text { dell'informazione finanziaria. } \\
\text { Nell'era dell'informazione, i } \\
\text { requisiti di mercato per i } \\
\text { laureati contabili stanno } \\
\text { cambiando progressivamente. }\end{array}$ \\
\hline $\begin{array}{l}\text { The Impact of } \\
\text { Information } \\
\text { Technology on The } \\
\text { Quality of Accounting } \\
\text { Information (SFAC NO } \\
8,2010 \text { ) }\end{array}$ & $\begin{array}{l}\text { Identificare se la } \\
\text { tecnologia ha avuto un } \\
\text { effetto (positivo } \\
\text { negativo) } \\
\text { applicazioni contabili e se } \\
\text { la tecnologia } \\
\text { dellinformazione nella } \\
\text { contabilità ha un effetto } \\
\text { sulla qualità dei rapporti } \\
\text { finanziari in Arabia } \\
\text { Saudita. }\end{array}$ & $\begin{array}{l}\text { La ricerca ha dimostrato che la } \\
\text { velocità e la flessibilità sono } \\
\text { l'effetto più tecnologico sulle } \\
\text { applicazioni contabili con } \\
\text { caratteristiche migliorate e } \\
\text { affidabili. }\end{array}$ \\
\hline $\begin{array}{l}\text { A Consultoria Contábil } \\
\text { como Oportunidade de } \\
\text { Negócios Profissionais }\end{array}$ & $\begin{array}{lr}\text { Condurre uno } & \text { studio } \\
\text { documentario } & \mathrm{e} \\
\text { bibliometrico } & \text { sulla }\end{array}$ & $\begin{array}{l}\text { La consulenza contabile } \\
\text { presenta un fattore di } \\
\text { rendimento }\end{array}$ \\
\hline
\end{tabular}

RC: 93887

Disponível em: https://www.nucleodoconhecimento.com.br/economia- 


\begin{tabular}{|c|c|c|}
\hline $\begin{array}{l}\text { - Um Est } \\
\text { Documental } \\
\text { Almeida et al (2018) }\end{array}$ & $\begin{array}{l}\text { onsulenza contabile nel } \\
\text { eriodo } 2006-2016 \text {. }\end{array}$ & $\begin{array}{l}\text { qualitativo per le aziende, } \\
\text { aumentando di conseguenza il } \\
\text { vantaggio competitivo per loro. }\end{array}$ \\
\hline $\begin{array}{l}\text { Consultoria } \\
\text { Organizacional como } \\
\text { um Instrumento de } \\
\text { Inovação em Arranjos } \\
\text { Produtivos Locais } \\
\text { Silva; Ribeiro e Silva } \\
\text { (2018). }\end{array}$ & $\begin{array}{l}\text { Analizzare in che modo le } \\
\text { pratiche di consulenza } \\
\text { organizzativa possono } \\
\text { innescare l'innovazione } \\
\text { negli accordi produttivi } \\
\text { locali (ACL). }\end{array}$ & $\begin{array}{l}\text { Le consulenze hanno fornito } \\
\text { innovazioni per la maggior } \\
\text { parte delle aziende, essendo } \\
\text { l'89\% nei processi, il } 77 \% \text { nella } \\
\text { gestione aziendale, il } 74 \% \text { nei } \\
\text { prodotti e il } 40 \% \text { nel marketing. }\end{array}$ \\
\hline $\begin{array}{l}\text { Planejamento } \\
\text { Estratégico: } \\
\text { Diagnóstico e Proposta } \\
\text { de Intervenção por } \\
\text { Meio de Consultoria }\end{array}$ & $\begin{array}{l}\text { Diagnosticare } \\
\text { presentare proposte di } \\
\text { intervento attraverso una } \\
\text { consulenzar che } \\
\text { contribuisca alla } \\
\text { formattazione di una } \\
\text { pianificazione strategica } \\
\text { per un'azienda attiva nel } \\
\text { business delle calzature e } \\
\text { degli accessori da donna } \\
\text { boutique. }\end{array}$ & $\begin{array}{l}\text { Attraverso un approccio di } \\
\text { consulenza esterna, una } \\
\text { visione } \\
\text { dell'organizzazione, } \\
\text { indispensabile adattare le } \\
\text { tecniche e gli strumenti al tipo } \\
\text { di business che viene } \\
\text { analizzato, nonché all'attività } \\
\text { svolta, alle dimensioni } \\
\text { dell'azienda e al volume delle } \\
\text { trattative. }\end{array}$ \\
\hline
\end{tabular}

Fonte: Paternità propria

Gli articoli selezionati hanno individuato ostacoli all'uso delle tecnologie da parte del contatore. La tabella 2 fornisce una sintesi di queste difficoltà dal riferimento dell'articolo selezionato.

RC: 93887

Disponível em: https://www.nucleodoconhecimento.com.br/economia- 
Tabella 2 - Ostacoli incontrati

\section{Titolo articolo, Riferimento}

No caminho da inovação: análise das capacidades de inovação de empresas contábeis diante das tecnologias digitais

Indústria 4.0 e avanços tecnológicos da área contábil: perfil, percepções e tecnologie expectativas dos profissionais

Características dos Diferentes La centralità del ruolo del contabile nei Modelos de Negócios Contábeis em modelli di business contabili tradizionali e Relação às Áreas da Contabilidade le risorse tecnologiche sono ancora a supporto in questo scenario.

As Contribuições da Contabilidade Adattarsi all'uso di nuovi strumenti Consultiva: Um Estudo de Caso/The tecnologici

Contributions of Advisory Accounting:

\section{A Case Study}

Impact of information technology on accounting line of works

The impact of information and communication technology (ICT) in the accounting system: advantages, applications, and challenges

The Impact of Information Technology on The Quality of Accounting Information (SFAC NO 8, 2010)

A Consultoria Contábil como Durata più lunga e allocazione di risorse Oportunidade de Negócios e costi di esecuzione maggiori.

RC: 93887

Disponível em: https://www.nucleodoconhecimento.com.br/economia- 
Profissionais - Um Estudo Documental

Consultoria Organizacional como um Manodopera.

Instrumento de Inovação em Arranjos

Produtivos Locais

Planejamento Estratégico: Diagnóstico Mancanza di conoscenza del software.

e Proposta de Intervenção por Meio de

Consultoria

Fonte: Paternità propria

Si osserva che ci sono diversi ostacoli evidenziati negli articoli quando si tratta dell'uso di tecnologie per la consulenza contabile. Questi ostacoli provengono principalmente dalle difficoltà di molti professionisti che non si adattano all'uso delle tecnologie nella loro routine, o perché il professionista non è adeguatamente qualificato o qualificato, o per paura dei cambiamenti tecnologici.

Schiavi et al (2020) sottolinea che le tecnologie dovrebbero essere viste come un supporto per puntare a buoni risultati soprattutto nelle consulenze, tuttavia, il commercialista dovrebbe essere consapevole dei progressi tecnologici che non sono lenti, il che consente al professionista di avere paura di utilizzare la tecnologia come supporto mirato alla consulenza.

Xavier; Carraro e Rodrigues (2020) affermano che la contabilità consultiva deve essere diretta dai mezzi tecnologici, perché favorisce più sicurezza, precisione e affidabilità nei dati trovati, tuttavia, perché ciò accada, spetta al commercialista essere consapevole dei cambiamenti nei progressi tecnologici e adattarsi all'intero sistema, portando al cliente tecnologie avanzate e di prima linea.

Schiavi e Behr (2020) e Angelim e Barreto (2020) riferiscono che una parte dei professionisti della contabilità ha ancora il loro dominio nella tradizionalità della scienza contabile, non consentendo la presenza totale di tecnologie per la migliore

RC: 93887

Disponível em: https://www.nucleodoconhecimento.com.br/economia- 
qualità nei servizi di consulenza contabile, il che riflette un significativo ritardo nella scoperta del processo decisionale.

Rende necessario che il professionista sia consapevole delle nuove tendenze tecnologiche per il miglioramento dell'azienda, un commercialista formato nell'uso delle tecnologie tende a ridurre i tempi per raggiungere l'equilibrio dell'azienda, oltre a riuscire a superare l'affidabilità nei dati raccolti.

Secondo Asuquo; Dan e Effiong (2020) la contabilità consulenziale offre l'opportunità di espandersi in tutti i settori della contabilità, sia in modo gestionale che finanziario, che determina il lavoro della consulenza è il motivo per cui si lavorerà sulla consulenza, tuttavia, il radicamento di contabilità tradizionale, crea un enorme ostacolo tra il commercialista e le tecnologie, dove il professionista si sente ancora più sicuro in presenza del cliente nel suo studio.

La scarsa conoscenza delle tecnologie e dei loro benefici permea ancora la vita di molti professionisti. La mancanza di formazione professionale, la mancanza di conoscenza dei programmi, la manodopera qualificata, tra gli altri ostacoli, consentono alle tecnologie di non entrare nella consulenza contabile e quindi favorire, in modo rapido, sicuro ed efficace, l'analisi dei dati e, di conseguenza, il processo decisionale (AL-SHAFEAY; AL_DUJAILI; AL-WATTAR, 2020; ALMEIDA et al., 2018; SILVA; RIBEIRO; SILVA, 2018; GAFURI, ROJO; MIURA, 2017).

Tuttavia, gli ostacoli non riguardano solo le difficoltà dei contabili nell'uso delle tecnologie, il malfunzionamento dei programmi, la lentezza di Internet, i virus nel computer possono influenzare la non idoneità del professionista al mezzo delle tecnologie per aiutare nella contabilità consultiva (AL NATOUR, 2020).

L'utilizzo di Software e Hardware richiede un'intensa dedizione del professionista per poter saper utilizzare gli strumenti e con questi metodi attuali che possono portare costi inferiori e maggiori benefici all'azienda. Considerando questi ostacoli nella loro

RC: 93887

Disponível em: https://www.nucleodoconhecimento.com.br/economia- 
complessità dell'ambiente tecnologico, identifica le potenzialità che le tecnologie possono portare alla consulenza contabile.

Tabella 3 - Possibilità di consulenza contabile e di utilizzazione delle tecnologie

\section{Titolo articolo, Riferimento}

No caminho da inovação: análise das capacidades de inovação de empresas contábeis diante das tecnologias digitais

Indústria 4.0 e avanços tecnológicos da área contábil: perfil, percepções e expectativas dos profissionais

Características dos Diferentes Modelos de Negócios Contábeis em Relação às Áreas da Contabilidade As Contribuições da Contabilidade Consultiva: Um Estudo de Caso/The Contributions of Advisory Accounting: A Case Study

Impact of information technology on accounting line of works

The impact of information and communication technology (ICT) in the accounting system: advantages, applications, and challenges

The Impact of Information
Technology on The Quality of Accounting Information (SFAC NO 8,

\section{Potenziale}

Utilizzo di diverse tecnologie digitali; agire pienamente online per portare maggiore accessibilità e agilità nella fornitura di servizi contabili

Agilità e guadagno di tempo. Comodità.

L'utilizzo dei mezzi tecnologici dell'utente come e-mail, telefono, tra gli altri per effettuare consulenza contabile.

Rapporto inscindibile tra tecnologia e contabilità consultiva promuovendo un supporto agile e di qualità ai suoi vari utenti.

Soddisfa il tuo pubblico interno ed esterno offrendo loro servizi di qualità e prestazioni su base continua e coerente.

Soddisfazione del cliente; Agilità; Affidabilità.

Velocità e flessibilità

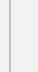

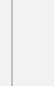

\section{RC: 93887}

Disponível em: https://www.nucleodoconhecimento.com.br/economia- 
2010)

A Consultoria Contábil como Rischioso ai risultati attesi del lavoro da Oportunidade de Negócios svolgere

Profissionais - Um Estudo

Documental

Consultoria Organizacional como um

Nuovi metodi di organizzazione del lavoro; Instrumento de Inovação em Arranjos Nuove tecniche per la gestione della Produtivos Locais conoscenza; Cambiamenti significativi nei rapporti con altre aziende

Planejamento Estratégico: Riduzione dei costi, controllo di gestione Diagnóstico e Proposta de con sviluppo per i programmi di acquisto. Intervenção por Meio de Consultoria

Fonte: Paternità propria

È evidente che la necessità di approfondire nuovi concetti e conoscenze è di fondamentale importanza per i professionisti della contabilità, per questo si osserva il potenziale delle tecnologie sulla consulenza contabile. La contabilità è considerata uno strumento fondamentale per supportare la crescita dell'azienda, tuttavia, la mancanza di conoscenza delle tecnologie da parte del commercialista rende queste conoscenze acquisite identificate nella tradizionalità dei ruoli.

Schiavi et al. (2020) menzionano diverse potenzialità che le tecnologie possono favorire la consulenza contabile, la citazione è: Uso di diverse tecnologie digitali; agire pienamente online per portare maggiore accessibilità e agilità nella fornitura di servizi contabili. Queste posizioni degli autori identificano l'importanza della contabilità e delle tecnologie che si muovono sempre l'una accanto all'altra, il che favorisce la fedeltà del commercialista ai propri clienti.

Xavier; Carraro e Rodrigues (2020) confermano con gli autori di cui sopra le potenzialità delle tecnologie per la consulenza contabile e rafforzano che oltre a

RC: 93887

Disponível em: https://www.nucleodoconhecimento.com.br/economia- 
questi collocamenti si può anche parlare di agilità, guadagno di tempo e convenienza sia dell'azienda che del commercialista.

Per Schiavi e Behr (2020) l'utilizzo di tecnologie per effettuare consulenza contabile non è solo legato a questioni di programmi informatici, telefono, social network, email, tra le altre tecnologie può aiutare il commercialista tradizionale a svolgere la sua consulenza senza necessariamente dover lavorare con programmi.

Secondo Angelim e Barreto (2020) le tecnologie hanno già la loro partecipazione alla contabilità consultiva, tuttavia, la presenza di programmi di facilitazione per la realizzazione della consulenza contabile ha portato a quella che viene chiamata tecnologia dell'innovazione, portando agilità e maggiore affidabilità nei dati presentati.

Secondo Asuquo; Dan ed Effiong (2020) e Al-Shafeay; Al Dujaili e Al-Wattar (2020), la contabilità consultiva mira a fornire le informazioni più affidabili sull'azienda e sui risultati che ottiene, la soddisfazione del cliente portando servizi di qualità e prestazioni è ciò che dimostra la differenza della presenza della tecnologia più avanzata per il commercialista.

Al Natour (2020) che le tecnologie sono veloci e flessibili, due condizioni essenziali per il lavoro del commercialista che nel suo tempo di consulenza diventa nemico del processo decisionale, poiché il mercato competitivo è sempre più esigente nei confronti delle aziende.

Secondo Almeida et al. (2018) le tendenze per aumentare il profitto di un'impresa hanno un impatto diretto sul contabile, il che intensifica la conoscenza contabile dell'assunzione di rischi. Le tecnologie agiscono al fine di garantire meno rischi per il commercialista e una migliore redditività per l'azienda, che consente al cliente e al proprietario dell'azienda un servizio di qualità e in un tempo agile.

RC: 93887

Disponível em: https://www.nucleodoconhecimento.com.br/economia- 
Silva; Ribeiro e Silva (2018) e Gafuri; Rojo e Miura (2017) sottolineano che oltre a fornire analisi accurate e sicure, le tecnologie forniscono anche metodi di organizzazione del lavoro, gestione della conoscenza, migliore rapporto con altre aziende, controllo dei costi, miglioramento del processo decisionale, tra le altre potenzialità volte a una maggiore produttività con costi inferiori rispetto alle tecniche tradizionali e manuali.

Si ritiene che le tecnologie combinate con la contabilità consultiva abbiano diverse potenzialità che consentono alla contabilità di avere il suo processo decisionale in modo che l'impresa non metta in difficoltà finanziarie o lasci il mercato competitivo, agendo in cima al problema evidenziato.

\section{CONSIDERAZIONI FINALI}

Gli ostacoli all'uso delle tecnologie nella contabilità consultiva sono ancora motivo di preoccupazione, considerando che il contabile dovrebbe avere come aiuto alla tecnologia per migliorare il proprio servizio e che la mancata accettazione dell'uso delle tecnologie può causare la squalifica e il discredito di questo professionista dalle sue funzioni.

II professionista contabile deve tenere a mente pratiche sostenibili per poter aumentare i benefici finanziari e operativi per le aziende, considerando che oltre all'elevato costo di acquisizione e manutenzione dell'intero processo di installazione tecnologica, ci sono spese per la qualificazione e la formazione professionale.

La crescente domanda di programmi per computer influenza direttamente la mancata accettazione di molti professionisti, questo può essere considerato in modo svantaggioso per la contabilità, poiché non esiste un unico sistema per svolgere le attività necessarie di cui la contabilità ha bisogno.

RC: 93887

Disponível em: https://www.nucleodoconhecimento.com.br/economia- 
Ma il potenziale che i progressi tecnologici apportano alla consulenza contabile sono immensi, e queste potenzialità possono generare impatti positivi per quanto riguarda l'accettazione dell'uso delle tecnologie per raggiungere gli obiettivi in contabilità. In questo contesto, è stato che le tecnologie facilitano la visione d'insieme del contatore, in cui la percezione di un minore margine di errore e l'accesso a tutti i dati di interesse che possono essere compilati e/o fatturati, trova nella tecnologia un alleato per svolgere un servizio di eccellenza.

Si ritiene pertanto che lo studio raggiunga il suo obiettivo elencato e si riferisca come un gap alla ricerca condotta sulla base della consulenza contabile in generale, che apre modi per idealizzare nuove ricerche estendendo questo tema a una forma più ristretta come la consulenza contabile finanziaria o anche manageriale.

\section{RIFERIMENTI}

ALMEIDA, Leonardo Soares Francisco de et al. A Consultoria Contábil como Oportunidade de Negócios Profissionais-um Estudo Documental. Pensar Contábil, v. 20, n. $72,2018$.

AL NATOUR, Jihad Rebhee Abdel Qader et al. The Impact of Information Technology on The Quality of Accounting Information (SFAC NO 8, 2010). Turkish Journal of Computer and Mathematics Education (TURCOMAT), v. 12, n. 13, p. 885-903, 2020.

AL-SHAFEAY, Karrar Mohammed; AL_DUJAILI, Mohammed Jawad; AL-WATTAR, Yasir Mohammed Ali. The impact of Information and Communication Technology (ICT) In the accounting system: advantages, applications, and challenges. PalArch's Journal of Archaeology of Egypt/Egyptology, v. 17, n. 6, p. 15814-15825, 2020.

ANGELIM, Vitória Ribeiro; BARRETO, Tayssa Vieira. As Contribuições da Contabilidade Consultiva: Um Estudo de Caso/The Contributions of Advisory

RC: 93887

Disponível em: https://www.nucleodoconhecimento.com.br/economia- 
Accounting: A Case Study. ID on line Revista de Psicologia, v. 14, n. 52, p. 317331, 2020.

ASUQUO, Akabom I.; DAN, Nicholas O.; EFFIONG, Glory T. Impact of information technology on accounting line of works. International Journal of Recent Technology and Engineering, v. 9, n. 2, p. 1572-1577, 2020.

DE ANDRADE SIMÕES, Maervelym Pâmella et al. Benefícios do uso da tecnologia Blockchain como instrumento para a auditoria contábil. Revista ambiente contábilUniversidade Federal do Rio Grande do Norte-ISSN 2176-9036, v. 13, n. 1, p. 3953, 2021.

ERCOLE, Flávia Falci; MELO, Laís Samara de; ALCOFORADO, Carla Lúcia Goulart Constant. Revisão integrativa versus revisão sistemática. Revista Mineira de Enfermagem, v. 18, n. 1, p. 9-12, 2014.

FERREIRA, Tiago Janes et al. Automação contábil: tecnologia em contabilidade sob a ótica da teoria institucional. XI Congresso ANPCONT. 3 a 6 de junho de 2017. Belo Horizonte, Minas Gerais.

GAFURI, Raquel Adriana Pin; ROJO, Cláudio Antonio; MIURA, Marcio Nakayama. Planejamento estratégico: diagnóstico e proposta de intervenção por meio de consultoria. Revista Capital Científico-Eletrônica (RCCe)-ISSN 2177-4153, v. 15, n. 1, p. 139-155, 2017.

MARION, JOSE CARLOS; RIBEIRO, OSNI MOURA. Introdução à contabilidade gerencial. Saraiva Educação SA, 2017. Disponível em: https://books.google.com.br/books?hl=pt-

$B R \& \mid r=\& i d=k D 1 n D w A A Q B A J \& o i=f n d \& p g=P T 5 \& d q=I n t r o d u \% C 3 \% A 7 \% C 3 \% A 30+\% C$ $3 \% \mathrm{~A} 0+$ contabilidade+gerencial. $+\&$ ots $=J j \mathrm{jSnq} 2 \mathrm{mD} 0 \&$ sig $=\mathrm{vcUiQXgi1UkNhLanfgWU67}$

RC: 93887

Disponível em: https://www.nucleodoconhecimento.com.br/economiaaziendale/attraverso-la-tecnologia 
QVgas\#v=onepage\&q=Introdu\%C3\%A7\%C3\%A30\%20\%C3\%A0\%20contabilidade\% 20gerencial. $\& f=$ false

SAMPAIO, Rosana Ferreira; MANCINI, Marisa Cotta. Estudos de revisão sistemática: um guia para síntese criteriosa da evidência científica. Brazilian Journal of Physical Therapy, v. 11, p. 83-89, 2007.

SCHIAVI, Giovana Sordi; BEHR, Ariel. Características dos diferentes modelos de negócios contábeis em relação às áreas da Contabilidade. REUNIR Revista de Administração Contabilidade e Sustentabilidade, v. 10, n. 3, p. 47-59, 2020.

SCHIAVI, Giovana Sordi et al. No caminho da inovação: análise das capacidades de inovação de empresas contábeis diante das tecnologias digitais. Revista Brasileira de Gestão de Negócios, v. 22, p. 381-405, 2020.

SILVA, Gustavo Oliveira et al. O impacto da tecnologia na profissão contábil sob perspectivas de pessoas com formação e/ou experiência profissional na área. Contribuciones a las Ciencias Sociales, n. 72, p. 3, 2020.

SILVA, Fabiana Ferreira; RIBEIRO, Ana Regina Bezerra; SILVA, Washington Ferreira. Consultoría Organizacional como um Instrumento de Inovação em arranjos produtivos locais. Revista Organizações em Contexto, v. 14, n. 27, p. 29-52, 2018.

VILLA, Patricia et al. O monólogo contábil: uma análise do uso da contabilidade gerencial em micro e pequenas empresas à luz do processo comunicacional segundo Bakhtin". Monografia para obtenção de título de mestrado em contabilidade. UFPR, 2012.

XAVIER, Leonardo Montes; CARRARO, Wendy Beatriz Witt Haddad; RODRIGUES, Ana Tércia Lopes. Indústria 4.0 e avanços tecnológicos da área contábil: perfil, percepções e expectativas dos profissionais. Contexto. Porto Alegre, RS. Vol. 20, n. 45 (maio/ago. 2020), p. 34-50, 2020.

RC: 93887

Disponível em: https://www.nucleodoconhecimento.com.br/economiaaziendale/attraverso-la-tecnologia 
Inviato: Luglio 2021.

Approvato: Luglio 2021.

RC: 93887

Disponível em: https://www.nucleodoconhecimento.com.br/economiaaziendale/attraverso-la-tecnologia 